$54^{\text {ème }}$ Congrès de la SFMBCB, 01004 (2011)

DOI: $10.1051 / \mathrm{sfmbcb} / 20115401004$

(c) Owned by the authors, published by EDP Sciences, 2011

\title{
La régénération des os longs en chirurgie orthopédique
}

\author{
Fabre Th \\ Pôle d'Orthopédie-Traumatologie, Unité membres, CHU, Bordeaux, France \\ thierry.fabre@chu-bordeaux.fr
}

Malgré les énormes progrès effectués ces 20 dernières années dans la compréhension de la physiologie de la régénération osseuse, la reconstitution du stock osseux pour des pertes de substance étendues reste un challenge thérapeutique dans un certain nombre de cas. La multiplicité des procédés proposés dans la littérature témoigne de la difficulté de la réparation d'une perte de substance osseuse. Des techniques variées sont rapportées avec leurs avantages et leurs inconvénients, souvent au prix de gestes chirurgicaux lourds, parfois répétés sur de nombreux mois et avec un résultat final pas toujours à la hauteur des espérances du praticien et du patient. L'existence de défauts de couverture des parties molles et d'infection osseuse sont indissociables car souvent associées aux pertes de substance osseuse. La prise en charge doit à ce titre être globale, s’intégrant dans une véritable stratégie de réparation des membres.

Le gold standard en matière de comblement des pertes de substance osseuse reste la greffe autologue de crête iliaque. Elle rassemble en son sein les 3 éléments indispensables à la régénération osseuse: des signaux correspondant aux facteurs de croissance de la lignée ostéocytaire, une matrice contenant ces signaux et qui servira de support à l'os qui va se former et, des cellules de l'hôte réceptrices du signal capable de se différencier en cellules osseuses et cellules endothéliales. Actuellement, aucun biomatériau hybride associant un substrat aussi proche soit il de la structure de l'os trabéculaire, des protéines inductrices de la formation osseuse et des cellules de l'hôte, qu'il s'agisse de cellules souches mésenchymateuses ou de cellules déjà différenciées, n’a donné chez le gros animal des résultats comparables à ceux de la greffe autologue. Néanmoins, ce prélèvement de crête iliaque ne permet pas de faire face à des pertes de substance osseuses circonférentielles supérieures à $3 \mathrm{~cm}$.

Dans ce contexte clinique, la technique des membranes induites rapportée par Masquelet et al. (2000) a permis d'intégrer dans une même stratégie chirurgicale, perte de substance osseuse, couverture des parties molles et infection osseuse. Cette technique permet de reconstruire des pertes de substance s'étendant jusqu'à $20 \mathrm{~cm}$. Le premier temps chirurgical est un temps d'exérèse - stabilisation osseuse avec mise en place d'une entretoise en ciment acrylique pour compenser de la perte de substance osseuse. Une membrane conjonctive va se développer autour de l'entretoise pour réaliser une véritable chambre biologique. Le second temps chirurgical qui intervient avant la fin du deuxième mois consiste à déposer, après l'ablation de la cale de ciment, une autogreffe spongieuse, fraiche, massive et morcelée, à l'intérieur de cette membrane conjonctive qui est soigneusement refermée. Masquelet et al. ont pu ainsi obtenir une consolidation osseuse en 8 mois pour 35 patients. Nous avons l'expérience de cette technique pour 30 patients opérés depuis 1999.

La percée récente des Bone MorphoProtéines (BMP) qui ont inondé le marché orthopédique telle une potion magique, n’est correctement évaluée et légalement proposée que dans 2 indications précises. Ainsi l'apport de BMP 2 améliore les taux de consolidation pour une fracture tibiale ouverte par rapport à la technique de référence (Govender et al. 2002). Par ailleurs, l'adjonction de BMP7 pour la cure chirurgicale d'une pseudarthrose du tibia donne des résultats identiques à la greffe osseuse autologue (Friedlaender et al. 2001). En pratique clinique, nous utilisons en routine la BMP7 après échec du traitement chirurgical d'une pseudarthrose. Masquelet sur 11 patients traités par la technique de membrane induite, avec adjonction de 3,5 mg de BMP7 à la greffe osseuse rapporte 3 absences de consolidation et des images radiologiques de résorption osseuse.

Les perspectives de recherche qui font l'objet de nombreux travaux (Khan et al. 2008, Patterson et al. 2008) dans la régénération osseuse s'orientent vers des biohybrides osseux associant des cellules ostéocompétentes et une matrice ostéoconductrice pour former un composite ostéogène. Les défis dans ce domaine sont nombreux. Le premier consiste a contourné la lourdeur de la technique d'expansion cellulaire peut être par l'intermédiaire de bioréacteurs. Le second consiste a assuré la survie cellulaire dans un environnement avasculaire et hypoxique, tel qu'il se présente en clinique humaine à la différence de la majorité des expérimentations animales rapportées dans la littérature. Enfin le troisième défi est représenté par le support qui doit procurer un environnement approprié pour stimuler la régénération osseuse et présenter des propriétés mécaniques contrôlables au cours du temps. Actuellement, un os fonctionnel peut être régénéré par thérapie cellulaire mais le potentiel ostéogène reste inférieur à celui de l'autogreffe osseuse.

This is an Open Access article distributed under the terms of the Creative Commons Attribution-Noncommercial License 3.0, which permits unrestricted use, distribution, and reproduction in any noncommercial medium, provided the original work is properly cited. 